\title{
COMPARISON OF SYMBOLIC AND ORDINARY POWERS OF IDEALS
}

\author{
By Melvin Hochster and Craig Huneke
}

\section{Introduction}

All given rings in this paper are commutative, associative with identity, and Noetherian. Recently, L. Ein, R. Lazarsfeld, and K. Smith [ELS] discovered a remarkable and surprising fact about the behavior of symbolic powers of ideals in affine regular rings of equal characteristic 0: if $h$ is the largest height of an associated prime of $I$, then $I^{(h n)} \subseteq I^{n}$ for all $n \geq 0$. Here, if $W$ is the complement of the union of the associated primes of $I, I^{(t)}$ denotes the contraction of $I^{t} R_{W}$ to $R$, where $R_{W}$ is the localization of $R$ at the multiplicative system $W$. Their proof depends on the theory of multiplier ideals, including an asymptotic version, and, in particular, requires resolution of singularities as well as vanishing theorems. We want to acknowledge that without their generosity and quickness in sharing their research this manuscript would not exist.

Our objective here is to give stronger results that can be proved by methods that are, in some ways, more elementary. Our results are valid in both equal characteristic 0 and in positive prime characteristic $p$, but depend on reduction to characteristic $p$. We use tight closure methods and, in consequence, we need neither resolution of singularities nor vanishing theorems that may fail in positive characteristic. For the most basic form of the result, all that we need from tight closure theory is the definition of tight closure and the fact that in a regular ring, every ideal is tightly closed. We note that the main argument

The authors were supported in part by grants from the National Science Foundation.

Version of July 25, 2001. 
here is closely related to a proof given in $[\mathrm{Hu}, 5.14-16, \mathrm{p} .45]$ that regular local rings in characteristic $p$ are UFDs, which proceeds by showing that Frobenius powers of height one primes are symbolic powers.

Our main results in all characteristics are summarized in the following theorem. Note that $I^{*}$ denotes the tight closure of the ideal $I$. The characteristic zero notion of tight closure used in this paper is the equational tight closure of [HH6] (see, in particular Definition (3.4.3) and the remarks in (3.4.4) of [HH6]). This is the smallest of the characteristic zero notions of tight closure, and therefore gives the strongest result. See $\S 3.1$ for a discussion of the Jacobian ideal $\mathcal{J}(R / K)$ utilized in part (c).

Theorem 1.1. Let $R$ be a Noetherian ring containing a field. Let $I$ be any ideal of $R$, and let $h$ be the largest height ${ }^{1}$ of any associated prime of $I$.

(a) If $R$ is regular, $I^{(h n+k n)} \subseteq\left(I^{(k+1)}\right)^{n}$ for all positive $n$ and nonnegative $k$. In particular, $I^{(h n)} \subseteq I^{n}$ for all positive integers $n$.

(b) If I has finite projective dimension then $I^{(h n)} \subseteq\left(I^{n}\right)^{*}$ for all positive integers $n$.

(c) If $R$ is finitely generated, geometrically reduced (in characteristic 0 , this simply means that $R$ is reduced) and equidimensional over a field $K$, and locally $I$ is either 0 or contains a nonzerodivisor (this is automatic if $R$ is a domain), then, with $J=\mathcal{J}(R / K)$, for every nonnegative integer $k$ and positive integer $n$, we have that $J^{n} I^{(h n+k n)} \subseteq\left(\left(I^{(k+1)}\right)^{n}\right)^{*}$ and $J^{n+1} I^{(h n+k n)} \subseteq\left(I^{(k+1)}\right)^{n}$. In particular, we have that $J^{n} I^{(h n)} \subseteq\left(I^{n}\right)^{*}$ and $J^{n+1} I^{(h n)} \subseteq I^{n}$ for all positive integers $n$.

These results, when specialized to the case where $R$ is regular, recover the cited result from $[\mathrm{ELS}]$.

The theorem above is a composite of Theorems 2.6, 3.7, and 4.4 below.

We note that by results ${ }^{2}$ of [Swsn] one expects, in many cases, to have results that assert that, given a fixed ideal $I$ in a Noetherian ring, for some choice of positive integer $h^{\prime}$ (independent of $n$ but depending on $I$ ) one has $I^{\left(h^{\prime} n\right)} \subseteq I^{n}$ for all positive integers $n$. What is not expected is the very simple choice of $h^{\prime}$ that one can make in a regular ring, and the extent to which it is independent of information about $I$. E.g., when $d=\operatorname{dim} R$

\footnotetext{
${ }^{1}$ The results stated here are all valid if one defines $h$ instead to be the largest analytic spread of $I R_{P}$ for any associated prime $P$ of $I$, which, in general, may be smaller: see Discussion 2.3

${ }^{2}$ E.g., it is shown in [Swsn] that if $I \subseteq J$ are ideals of a Noetherian ring, and we let $I: J^{\infty}=\bigcup_{t} I: J^{t}$, then if the $I$-adic filtration is equivalent to the $I^{n}: J^{\infty}$ filtration, there exists an integer $h^{\prime}$ such that for all $n, I^{h^{\prime} n}: J^{\infty} \subseteq I^{n}$.
} 
is finite, then with $a=d$ one has that $I^{(a n)} \subseteq I^{n}$ for all ideals $I$ (if $R$ is local one has this for all unmixed $I$ with $a=\max \{d-1,1\}$ - one does not have to worry about letting $h=d$, since for the maximal ideal one has that ordinary and symbolic powers coincide).

We conclude this introduction by sketching the proof of Theorem 1.1(a) for regular domains in characteristic $p>0$ when $k=0$ in the special case where $I$ is a radical ideal. The proof is very simple and brief in this case, and we hope that this argument will help the reader through the complexities of the rest of this paper. Suppose that $I \neq(0)$ is a radical ideal, and let $h$ be the largest height of any minimal prime. If $u \in I^{(h n)}$, then for every $q=p^{e}$ we can write $q=a n+r$ where $a \geq 0$ and $0 \leq r \leq n-1$ are integers. Then $u^{a} \in I^{(h a n)}$ and $I^{h n} u^{a} \subseteq I^{h r} u^{a} \subseteq I^{(h a n+h r)}=I^{(h q)}$. We now come to a key point: we can show that $(*) I^{(h q)} \subseteq I^{[q]}$. To see this, note that because the Frobenius endomorphism is flat for regular rings, $I^{[q]}$ has no associated primes other than the minimal primes of $I$ (cf. Lemma $(2.2 \mathrm{~d})$ ), and it suffices to check $(*)$ after localizing at each minimal prime $P$ of $I$. But after localization, $I$ has at most $h$ generators, and so each monomial of degree $h q$ in these generators is a multiple of the $q$ th power of at least one of the generators. This completes the proof of $(*)$. Taking $n$th powers gives that $I^{h n^{2}} u^{a n} \subseteq\left(I^{[q]}\right)^{n}=\left(I^{n}\right)^{[q]}$, and since $q \geq a n$, we have that $I^{h n^{2}} u^{q} \subseteq\left(I^{n}\right)^{[q]}$ for fixed $h$ and $n$ and all $q$. Let $d$ be any nonzero element of $I^{h n^{2}}$. The condition that $d u^{q} \in\left(I^{n}\right)^{[q]}$ for all $q$ says precisely that $u$ is in the tight closure of $I^{n}$ in $R$. But in a regular ring, every ideal is tightly closed (cf. [HH2, Th. (4.4)]), and so $u \in I^{n}$, as required.

\section{The regular case in characteristic $p$}

Discussion 2.1. We recall some terminology and notation. $R^{\circ}$ denotes the complement of the union of the minimal primes of $R$, and so, if $R$ is reduced, $R^{\circ}$ is simply the multiplicative system of all nonzerodivisors in $R$. We shall write $\boldsymbol{F}^{e}$ (or $\boldsymbol{F}_{R}^{e}$ if we need to specify the base ring) for the Peskine-Szpiro or Frobenius functor from $R$-modules to $R$-modules. This is a special case of the base change functor from $R$-modules to $S$-modules that is simply given by $S \otimes_{R}$ _: in the case of $\boldsymbol{F}^{e}$, the ring $S$ is $R$, but the map $R \rightarrow R$ that is used for the algebra structure is the $e$ th iteration $F^{e}$ of the Frobenius endomorphism: $F^{e}(r)=r^{p^{e}}$. We shall use the notation ${ }^{(e)} R$ for $R$ viewed as an $R$-algebra via the homomorphism $F_{R}^{e}: R \rightarrow R$.

In particular, if $M$ is given as the cokernel of the map represented by a matrix $\left(r_{i j}\right)$, then $\boldsymbol{F}^{e}(M)$ is the cokernel of the map represented by the matrix $\left(r_{i j}^{p^{e}}\right)$. Unless otherwise 
indicated, $q$ denotes $p^{e}$ where $e \in \mathbb{N}$. For $q=p^{e}, \boldsymbol{F}^{e}(R / I) \cong R / I^{[q]}$, where $I^{[q]}$ denotes the ideal generated by the $q$ th powers of all elements of $I$ (equivalently, of generators of $I)$. Note that $\boldsymbol{F}^{e}$ preserves both freeness and finite generation of modules, and is exact precisely when $R$ is regular (cf. [Her], [Kunz]).

Lemma 2.2 (Peskine-Szpiro). Let $R$ be a Noetherian ring of characteristic $p$, and $M$ be a finitely generated $R$-module of finite projective dimension over $R$. Then:

(a) For all $i \geq 1, \operatorname{Tor}_{i}^{R}\left(M,{ }^{(e)} R\right)=0$.

(b) If $R$ is local and one applies $\boldsymbol{F}^{e}$ to a minimal free resolution of $M$, one obtains a minimal free resolution of $\boldsymbol{F}^{e}(M)$. In particular, $\operatorname{pd}_{R} M=\operatorname{pd}_{R} \boldsymbol{F}^{e}(M)$.

(c) For all $e \geq 1$, the set of associated primes of $M$ is the same as the set of associated primes of $\boldsymbol{F}^{e}(M)$.

(d) In particular, if $R$ is regular, so that $\boldsymbol{F}^{e}$ is flat, then the conclusions of (b) and (c) are valid for every finitely generated $R$-module.

Proof. We refer to [PS] for part (a). Part (b) is well-known and is immediate from (a). Part (c) is likewise well-known, but we mention that it reduces to the local case by localization at a given prime, and so it reduces to checking that the maximal ideal of $R$ is associated to $M$ if and only if it is associated to $\boldsymbol{F}^{e}(M)$. But when $M$ is a module of finite projective dimension, $m$ is associated to $M$ if and only if $\operatorname{pd}_{R} M=\operatorname{depth} R$.

We refer the reader to $[\mathrm{PS}],[\mathrm{Her}]$, and [Kunz] for related results.

\section{Discussion 2.3: integral dependence of ideals, analytic spread, and minimal} reductions. Recall that an element $r$ of a ring $R$ is integrally dependent on an ideal $I$ if there is an integer $t \geq 1$ and an equation of the form $r^{t}+i_{1} r^{t-1}+\cdots+i_{k} r^{k}+\cdots+i_{t-1} r+i_{t}=$ 0 , where $i_{k} \in I^{k}, 1 \leq k \leq t$. The elements of the ring $R$ integrally dependent on $I$ form an ideal $J \supseteq I$, the integral closure of $I$ in $R$. We refer the reader to [L1], §5 of [HH2], and [NR1-2] for more detailed information about integral dependence and analytic spread.

(a) In a Noetherian local ring $(R, m, K)$ with maximal ideal $m$ and residue field $K$, the analytic spread $\mathrm{a}(I)$ of an ideal $I \subseteq m$ is the Krull dimension of the ring

$$
K \otimes_{R} \operatorname{gr}_{I} R \cong K \oplus I / m I \oplus I^{2} / m I^{2} \oplus \cdots \oplus I^{k} / m I^{k} \oplus \cdots
$$

The analytic spread is a lower bound on the least number of generators of an ideal $I_{0} \subseteq I$ such that $I$ is integrally dependent on $I_{0}$. If $K$ is infinite, there always is an ideal $I_{0}$ with 
$\mathrm{a}(I)$ generators such that $I$ is integral over $I_{0}$, and such an ideal $I_{0}$ is called a minimal reduction of $I$. When $K$ is infinite, one may find generators for a minimal reduction $I_{0}$ of $I$ by simply taking a linear homogeneous system of parameters for $K \otimes_{R} \operatorname{gr}_{I} R$, say $f_{1}, \ldots, f_{\mathrm{a}} \in I / m I \cong\left[K \otimes_{R} \operatorname{gr}_{I} R\right]_{1}$, and lifting the $f_{j}$ to elements of $I$.

Note that the analytic spread of $I$ is bounded both by the number of generators of $I$ and by the Krull dimension of $R$.

(b) If $I$ is an ideal of $R$ and $t$ is an indeterminate over $R$, then the associated primes of $I R[t]$ are those of the form $Q=P R[t]$ where $P$ is an associated prime of $I$. For this $Q$ the analytic spread of $I R[t]_{Q}$ is the same as the analytic spread of $I R_{P}$. Thus, the maximum analytic spread after localization at an associated prime is the same for $I R[t]$ in $R[t]$ as it is for $I$ in $R$. Moreover, the symbolic powers of $I R[t]$ are the expansions of the symbolic powers of $I$.

(c) If $S$ is flat over $R$ then the maximum analytic spread of $I S$ after localizing at an associated prime in $S$ is at most what it was for $I$ in $R$. To see this, first note that by replacing $R \rightarrow S$ by $R[t] \rightarrow S[t]$ and $I$ by $I R[t]$, we may assume without loss of generality that the residue fields of the local rings of associated primes of $I$ in $R$ are infinite. We return to the original notation. By Proposition 15 in Section IV B.4. of [Se], $Q$ is an associated prime of $I S$ if and only if it is an associated prime of (0) in $S / P S$ for some associated prime $P$ of $I$. But $S / P S$ is flat and, hence, torsion-free over the domain $R / P$, which implies that $Q$ lies over $P$. Thus, we have a map $R_{P} \rightarrow S_{Q}$. If the analytic spread of $I R_{P}$ is $h$, it is integral over an ideal with $h$ generators. But then $I S_{Q}$ is integral over the expansion of the same ideal, and the result follows.

(d) We recall also that in a Noetherian ring $R, I$ is integrally dependent on $I_{0}$ if and only if there exists an integer $k$ such for all positive integers $n, I^{k+n}=I^{k} I_{0}^{n}$. In particular, it then follows that $I^{k+n} \subseteq I_{0}^{n}$ for all positive integers $n$.

Part (b) of the next result plays a critical role in the proofs of our theorems. It is closely related to the Briançon-Skoda theorem, and related results were used to prove a tight closure form of the Briançon-Skoda theorem in $\S 5$ of [HH2]. In fact, our first proofs of some of the results here made use of the Briançon-Skoda theorem in a sharpened form ${ }^{3}$

\footnotetext{
${ }^{3}$ To be precise, if $I$ is integral over an ideal with at most $h$ generators in a Noetherian domain of characteristic $p>0$, then the integral closure of $I^{h}$ is contained in $I^{+}$. Here, if $R^{+}$denotes the integral closure of $R$ is an algebraic closure of its fraction field (which is unique up to non-unique isomorphism),
} 
given in [HH5], Theorem (7.1), that uses plus closure instead of tight closure, together with the fact that plus closure commutes with localization.

Lemma 2.4. Let $R$ be a ring.

(a) If $I=\left(u_{1}, \ldots, u_{h}\right)$ then for all integers $t \geq 1$ and $k \geq 0$,

$$
I^{h t+k t-h+1} \subseteq\left(u_{1}^{t}, \ldots, u_{h}^{t}\right)^{k+1} .
$$

In particular, $I^{h t+k t} \subseteq\left(u_{1}^{t}, \ldots, u_{h}^{t}\right)^{k+1}$. Hence, if $R$ has prime characteristic $p>0$ and $q=p^{e}$ is a power of $p$, then $I^{h q+k q} \subseteq\left(I^{[q]}\right)^{k+1}=\left(I^{k+1}\right)^{[q]}$.

(b) (Key Lemma) Let $R$ be Noetherian of positive prime characteristic p. Suppose that $I$ is an ideal of $R$, that $W$ is the complement of the union of the associated primes of $I$, and that ${ }^{W}$ indicates the result of expanding an ideal of $R$ to $R_{W}$ and then contracting it to $R$. Suppose that for every associated prime $P$ of $I, I R_{P}$ has analytic spread at most $h$ in $R_{P}$. Then there is a fixed positive integer s (depending on I) with the following property:

For all choices of integers $n \geq 0, q=p^{e}$, and $k \geq 0$, we have that if $u \in I^{(h n+k n)}$ then

$$
I^{s+(h+k)(n-1)} u^{\lfloor q / n\rfloor} \in\left(\left(I^{(k+1)}\right)^{[q]}\right)^{W},
$$

where $\lfloor q / n\rfloor$ denotes the integer part of $q / n$. If $R$ is regular or if $I$ has finite projective dimension and $k=0$, the superscript ${ }^{W}$ can be omitted.

Proof. Consider any monomial $u_{1}^{b_{1}} \cdots u_{h}^{b_{h}}$ in the $u_{j}$ in which the sum of the exponents, $b_{1}+\cdots+b_{h}$, is at least $h t+k t-h+1$. Write each $b_{j}=a_{j} t+c_{j}$ where $a_{j}$ is a positive integer and $0 \leq c_{j} \leq t-1$. Then it suffices to prove that the sum of the $a_{j}$ at least $k+1$, for then the original monomial is a multiple of $\left(u_{1}^{t}\right)^{a_{1}} \cdots\left(u_{h}^{t}\right)^{a_{h}} \in\left(u_{1}^{t}, \ldots, u_{h}^{t}\right)^{k+1}$. But, otherwise, we have that the sum of the $a_{k}$ is at most $k$, which means that the sum of the $b_{j}$ is at most $k t+h(t-1)<h t+k t-h+1$, a contradiction. (A slightly weaker version is proved in a parenthetical comment near the bottom of p. 45 of [HH2].) The remaining statements in (a) are immediate.

then $I^{+}=I R^{+} \cap R$. It is known that $I \subseteq I^{+} \subseteq I^{*}$, while equality for ideals generated by part of a system of parameters in an excellent local domain is established in [Sm1]. Because the formation of $R^{+}$commutes with localization at a multiplicative system, plus closure commutes with localization. We refer the reader to [HH4-5] and [Sm1] for further discussion of $R^{+}$. 
For part (b), first note that the issues are unaffected by adjoining an indeterminate $t$ to the ring $R$ and replacing $I$ by $I R[t]$. A choice of $s$ that works for $I R[t]$ and $R[t]$ will work for $I$ and the original ring $R$ : the associated primes of $I R[t]$ are simply those of the form $P R[t]$, where $P$ is an associated prime of $I$, and if $W^{\prime}$ is the complement of the union of the associated primes of $I R[t]$ we have that $R[t]_{W^{\prime}}$ is faithfully flat over $R_{W}$. Moreover, for every $P$, the analytic spread of $I R[t]_{P R[t]}$ is the same as the analytic spread of $I R_{P}$.

Thus, we may assume without loss of generality that the residue field of each of the rings $R_{P}$ is infinite when $P$ is an associated prime of $I$, and it follows that for each associated prime $P_{i}$ of $I$ we can choose an ideal $J_{i} \subseteq I$ with at most $h$ generators such that $I_{P_{i}}$ is integral over $\left(J_{i}\right)_{P_{i}}$. By 2.3(d) there is a positive integer $s_{i}$ such that $I_{P_{i}}^{s_{i}+N} \subseteq\left(J_{i}\right)_{P_{i}}^{N}$ for all positive integers $N$. Let $s$ be the maximum of the $s_{i}$. Write $q=a n+r$ with $0 \leq r \leq n-1$. To prove that $I^{s+(h+k)(n-1)} u^{a} \in\left(\left(I^{(k+1)}\right)^{[q]}\right)^{W}$ it suffices to prove that whenever $P=P_{i}$ is an associated prime of $I$, we have that

$$
I^{s+(h+k)(n-1)} u^{a} R_{P} \subseteq\left(\left(I^{(k+1)}\right)^{[q]}\right) R_{P} .
$$

Let $J=J_{i}$. Since $R_{P}$ contains $W^{-1}$, after localization at $P$ the symbolic and ordinary powers of $I$ are the same. But then (recall that $q=a n+r$ with $0 \leq r \leq n-1$ ), we have that

$$
I_{P}^{s+(h+k)(n-1)} u^{a} \subseteq I_{P}^{s+(h+k) r+(h+k) a n} \subseteq I_{P}^{s+(h+k)(a n+r)} \subseteq J_{P}^{(h+k) q}
$$

(the last inclusion holds by the choice of $s$ ). But $J_{P}^{(h+k) q} \subseteq\left(J_{P}^{k+1}\right)^{[q]}$ by part (a), and this is clearly contained in $\left.\left(I_{P}^{(k+1)}\right)^{[q]}\right)$, as required.

The omission of $W$ when $R$ is regular is justified by the fact that $I^{(k+1)}$ has the property that no element of $W$ is a zerodivisor on $R / I^{(k+1)}$, and, since the Frobenius endomorphism is flat, the elements of $W$ are also nonzerodivisors on $R /\left(I^{(k+1)}\right)^{[q]}$ for all $q$ by Lemma 2.2(d). If instead $k=0$ and $I$ has finite projective dimension we may apply Lemma 2.2(c) instead.

We shall say that an ideal $I$ of a Noetherian ring $R$ is locally generically free if for every prime ideal $P$ of $R, I R_{P}$ is either (0) or contains a nonzerodivisor. When $R$ is local with total quotient ring $T$, this is equivalent to requiring that $I T$ be free (of rank 0 or 1, necessarily). Notice that ideals of finite projective dimension are automatically locally generically free. This is well-known, but we indicate a brief argument. The point is that in the local case one has a free resolution, and so whenever one has a localization such that 
the ideal is projective ( $\equiv$ free), the rank is the same as the alternating sum of the ranks of the free modules in the finite free resolution. Once one tensors with the total quotient ring (of the local ring) one has a semilocal ring with all maximal ideals of depth 0 . By the Auslander-Buchsbaum theorem, all modules of finite projective dimension are now locally free. By the remarks above the rank is constantly 0 or 1 , so that the ideal has become either (0) or free of rank one.

Lemma 2.5. Let $R$ be a Noetherian ring.

(a) If $R$ is Noetherian and $I$ is an ideal containing a nonzerodivisor, then I is generated by the nonzerodivisors in $I$.

(b) If $R$ is Noetherian local and $I \neq 0$ has finite projective dimension, then it contains a nonzerodivisor. I.e., if $R$ is any Noetherian ring and $I$ has finite projective dimension, then $I$ is locally generically free.

(c) If $R$ is Noetherian with Spec $R$ connected and $I \neq 0$ is locally generically free, then it contains a nonzerodivisor.

Proof. For part (a), let $I_{0} \subseteq I$ be the ideal generated by all nonzerodivisors in $I$. Then $I$ is contained in the union of $I_{0}$ and the associated primes of (0) in the ring. Since $I$ is not contained in any associated prime of (0), we must have that $I \subseteq I_{0}$, and so $I=I_{0}$. Part (b) was established in the discussion preceding the statement of the lemma. Finally, to prove (c), let $S$ be the set of primes $P$ such that $I R_{P}$ contains a nonzerodivisor and let $T$ be the set of primes $P$ such that $I R_{P}$ is zero. Then $\operatorname{Spec} R$ is the disjoint union of these two sets. Both have the property that if $P \subseteq Q$ and $Q$ is in the set, then $P$ is in the set. It follows that if $P \subseteq Q$ and $P$ is in one of these sets, then $Q$ is in the same set. Thus, both sets are Zariski closed. Since Spec $R$ is connected, one of these sets is empty, and since $I \neq(0)$, we have that $T$ is empty. Then $I$ is not contained in any associated prime $P$ of (0) (or its localization $I R_{P}$ would consist entirely of zerodivisors). Hence, there is an element of $I$ not in any associated prime of (0).

We are now ready to prove one of our main results.

Theorem 2.6. Let I be ideal of a Noetherian ring of positive prime characteristic p. Let $h$ be the largest height of any associated prime of I (or let $h$ be the largest analytic spread of $I R_{P}$ for $P$ an associated prime of $\left.I\right)$. Then, if $R$ is regular, $I^{(h n)} \subseteq I^{n}$ for every positive integer $n$, while if I has finite projective dimension, $I^{(h n)} \subseteq\left(I^{n}\right)^{*}$ for every positive integer 
$n$.

If $R$ is regular one has more generally that for every nonnegative integer $k, I^{(h n+k n)} \subseteq$ $\left(I^{(k+1)}\right)^{n}$ for every positive integer $n$.

Proof. Since $I$ has finite projective dimension (this is automatic if the ring is regular), we may apply Lemma 2.4. If $R$ is a product we may consider the problem for the various factors separately, and so we may assume without loss of generality that $\operatorname{Spec} R$ is connected. If $I=(0)$ there is nothing to prove. Otherwise, by Lemma 2.5(c), $I$ contains a nonzerodivisor.

We handle all cases of the theorem at once by assuming either that $R$ is regular or that $I$ has finite projective dimension and that $k=0$. Choose $s$ as in the Key Lemma 2.4(b). For every $q=p^{e}$ we may write $q=a n+r$, where $a$ is a nonnegative integer and $0 \leq r \leq n-1$. Now, $u \in I^{(h n+h k)}$ implies, by Lemma 2.4(b), that $I^{s+(h+k)(n-1)} u^{a} \subseteq\left(I^{(k+1)}\right)^{[q]}$ (note that in both cases the superscript $W$ is not needed) and we may raise both sides to the $n$th power to get

$$
I^{s n+(h+k)(n-1) n} u^{a n} \subseteq\left(\left(I^{(k+1)}\right)^{n}\right)^{[q]} .
$$

We may multiply by $u^{r}$, and so abbreviating $b=s n+(h+k)(n-1) n$ we have that $I^{b} u^{q} \subseteq$ $\left(\left(I^{(k+1)}\right)^{n}\right)^{[q]}$ for all $q$. Since $I$ contains a nonzerodivisor, so does $I^{b}$ : call it $d$. Notice that $b$, and, hence, $I^{b}$, does not depend on $q$. We therefore have that $d u^{q} \in\left(\left(I^{(k+1)}\right)^{n}\right)^{[q]}$ for all $q$. Thus, $u \in\left(\left(I^{(k+1)}\right)^{n}\right)^{*}$. Since every ideal is tightly closed in case the ring is regular, the proof is complete in all cases.

Remark 2.7. The result above is also valid for ideals $I$ in Noetherian $\operatorname{rings} R$ if $V(I)$ is disjoint from the singular locus of $R$, and the singular locus is closed. (If $I_{1}$ defines the singular locus then $I+I_{1}=R$. Choose $f \in I_{1}$ so that it is a unit in $R / I$. Then it is also a unit modulo any any ideal containing a power of $I$. It follows that $R / I^{\prime} \cong R_{f} / I^{\prime}$ whenever

$I^{\prime}=I^{n}, I^{(h n+k n)}$, or $\left(I^{(k+1)}\right)^{n}$, and the result is immediate from this observation and the fact that we may apply Theorem 2.6 to the regular ring $R_{f}$.) Precisely the same observation holds in the equal characteristic 0 case, making use of Theorem 4.4 instead.

\section{Singular affine algebras in positive characteristic}

The results of this section depend heavily on the fact that, in positive characteristic, the elements of the Jacobian ideal can be used not only as test elements, but also have 
the property that their $q$ th powers "multiply away" the effects of embedded components of $q$ th bracket powers of unmixed ideals (these do not occur in the regular case): a precise statement is given in Lemma 3.6. Statements of this sort depend heavily in turn on the Lipman-Sathaye Jacobian theorem.

Discussion 3.1: the Jacobian ideal. Let $A$ be a reduced Noetherian ring with total quotient ring $T=\mathcal{T}(A)$, so that $T$ is a finite product of fields. Let $R$ be a finitely generated $A$-algebra such that $R$ is torsion-free over $A$ (i.e., nonzerodivisors of $A$ are nonzerodivisors on $R$ ) and such that $T \otimes_{A} R$ is equidimensional of dimension $d$ and geometrically reduced. We then define the Jacobian ideal $\mathcal{J}(R / A)$ as follows. Choose a finite presentation of $R$ over $A$, say $R \cong A\left[x_{1}, \ldots, x_{n}\right] /\left(f_{1}, \ldots, f_{m}\right)$, and let $\mathcal{J}(R / A)$ denote the ideal of $R$ generated by the images of the $n-d$ size minors of Jacobian matrix $\left(\partial f_{j} / \partial x_{i}\right)$. An important case is where $A=K$ is a field.

We note the following easy facts:

(1) $\mathcal{J}(R / A)$ is independent of the choice of presentation. E.g., if one changes the set of generators $f_{j}$ of the denominator ideal, it suffices to compare the result from each set of generators with the union. By induction, one only needs to see what happens with one additional generator. The calculation is then very easy. If one has two different presentations one can put them together (think of the two sets of variables as disjoint and independent). Thus, one need only compare Jacobians when one uses some extra generators to give a presentation, and, by induction, it suffices to consider the case of one extra generator. But then the denominator ideal has the form $f_{1}, \ldots, f_{m}, y-g\left(x_{1}, \ldots, x_{n}\right)$ where $y$ is a new variable and $g$ maps to the extra generator in $R$. Again, the calculation is now easy.

(2) Let $A \rightarrow B$ be any map such that $B$ is reduced and flat over $A$, and let $R_{B}=$ $B \otimes_{A} R$. Then $\mathcal{J}\left(R_{B} / B\right)$ is defined, and $\mathcal{J}\left(R_{B} / B\right)=\mathcal{J}(R / A) R_{B}$. Note that there is an induced map of total quotient rings $T \rightarrow T^{\prime}$, and it follows easily that $T^{\prime} \otimes R_{B}$ is geometrically reduced and equidimensional of dimension $d$. Also note that $R_{B}$ is torsion-free over $B: R$ is a directed union of finitely generated $A$-submodules that are embeddable in free $A$-modules, and since $B$ is flat over $A$ this property is preserved by $B \otimes_{A} \_$.

(3) In particular, we may apply (2) whenever $B$ is any localization of $A$, or if $A$ is a field and $B$ is any extension field. 
(4) If $\mathcal{J}(R / A)$ is defined and $S$ is the localization of $R$ at one element $f$, i.e., $S=R_{f}$, then $\mathcal{J}(S / A)$ is defined and equal to $\mathcal{J}(R / A) S_{f}$. Note that if we have a presentation of $R$ such that $g\left(x_{1}, \ldots, x_{n}\right)$ maps to $f$, then we get a presentation of $S$ by using one additional variable $y$ and one additional generator for the denominator ideal, $y g-1$, and the calculation is then routine.

(5) Given ring extensions $A \rightarrow R$ and $R \rightarrow S$ such that $\mathcal{J}(R / A)$ and $\mathcal{J}(S / R)$ are defined with $\mathcal{T}(A) \otimes_{A} R$ of dimension $d$ and $\mathcal{T}(R) \otimes_{R} S$ of dimension $d^{\prime}$, then $\mathcal{J}(S / A)$ is defined, $\mathcal{T}(A) \otimes_{A} S$ has dimension $d+d^{\prime}$, and $\mathcal{J}(S / A) \supseteq \mathcal{J}(S / R) \mathcal{J}(R / A)$. (Certainly, $S$ is torsion-free over $A$. The statements about being reduced or geometrically reduced and about dimension can be checked after tensoring with $T$, and we may, in fact, assume that $A=T$ is a field. The verifications are now straightforward. For example, the statement about dimension can be verified, for each component of $S$, using the additivity of transcendence degree. For the statement about products of Jacobians we can take a presentation of $S$ over $A$ of the form

$$
A\left[x_{1}, \ldots, x_{n}, y_{1}, \ldots, y_{t}\right] /\left(f_{1}(\underline{x}), \ldots, f_{m}(\underline{x}), g_{1}(\underline{x}, \underline{y}), \ldots, g_{s}(\underline{x}, \underline{y})\right)
$$

where $\underline{x}=x_{1}, \ldots, x_{n}$ and $\underline{y}=y_{1}, \ldots, y_{t}$. Here, we can assume that

$$
A\left[x_{1}, \ldots, x_{n}\right] /\left(f_{1}(\underline{x}), \ldots, f_{m}(\underline{x})\right)
$$

is a presentation of $R$ over $A$ (let $M$ be the Jacobian matrix with entries mapped to $R$ ), and that the images of the $g_{k}$ in $R\left[y_{1}, \ldots, y_{t}\right]$ may be used to give a presentation of $S$ over $R$ (let $N$ be the corresponding Jacobian matrix with entries mapped to $S$ ). Then the Jacobian matrix for $S$ over $A$ for this presentation with entries mapped to $S$ has the block form $\left(\begin{array}{cc}M & U \\ 0 & N\end{array}\right)$. Given $n-d$ rows and columns of $M$ (corresponding to the choice of a minor) and $t-d^{\prime}$ rows and columns of $N$, we get $(n+t)-\left(d+d^{\prime}\right)$ rows and columns of this block matrix, and the determinant of the minor they determine is the product of the determinants of minors chosen from $M$ and $N$.)

Discussion 3.2: test elements. An element $c \in R^{\circ}$ is called a test element if, whenever $M$ is a finitely generated $R$-module and $N \subseteq M$ is a submodule, then $u \in M$ is in the tight closure of $N$ if and only if for all $q=p^{e}, c u^{q} \in N^{[q]}$ (the image of $\boldsymbol{F}^{e}(N) \rightarrow \boldsymbol{F}^{e}(M)$ ). Thus, 
if the ring has a test element, it "works" in any tight closure test where some choice of $c \in R^{\circ}$ "works." Test elements are also characterized as the elements of $R^{\circ}$ that annihilate $N^{*} / N$ for all submodules $N$ of all finitely generated modules $M$.

A test element is called locally stable if its image in every local ring of $R$ is a test element (this implies that it is a locally stable test element in every localization of $R$ at any multiplicative system). A test element is called completely stable if its image in the completion of each local ring of $R$ is a test element: a completely stable test element is easily seen to be locally stable. We refer the reader to [HH1], [HH2, $\S 6$ and $\S 8$ ], and [HH3, $\S 6]$ for more information about test elements, For the moment we simply want to note that if $R$ is any reduced ring essentially of finite type over an excellent local ring, then $R$ has a test element. In fact, if $c$ is any element of $R^{\circ}$ such that $R_{c}$ is regular (and such elements always exist if $R$ is excellent and reduced), then $c$ has a power that is a completely stable test element. This follows from Theorem (6.1a) of [HH3].

3.3 Discussion. When $R$ is a reduced ring of positive prime characteristic $p$ and $q=p^{e}$, we write $R^{1 / q}$ for the unique reduced $R$-algebra obtained by adjoining $q$ th roots for all elements of $R$. Thus, there is a commutative diagram:

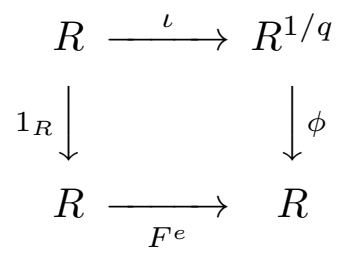

where $\iota$ is an inclusion map and $\phi(s)=s^{q}$. We write $R^{\infty}$ for the increasing union of the rings $R^{1 / q}$. The following result is a variant of the results of $\S 1.5$ of [HH6]: the differences from what is done in [HH6] are discussed in the proof.

Theorem 3.4. Let $R$ be a geometrically reduced equidimensional affine algebra of dimension d over a field $K$ of positive prime characteristic $p$. Let $t$ be an indeterminate over $K$, let $L=K(t)$, and let $R_{L}=L \otimes_{K} R$. Let $J^{\prime}=J_{R_{L} / L}$ be the Jacobian ideal of $R_{L}$ over $L$, which is evidently $\mathcal{J}(R / K) R_{L}$. There are always elements of $\mathcal{J}(R / K)$ in $R^{\circ}$ (so that $\mathcal{J}(R / K)$ is generated by such elements), and these are completely stable test elements for $R$. Moreover, $J^{\prime}$ is generated by elements $c$ such that

(*) There is a regular subring $A$ of $R$ (depending on $c$ ), in fact, a polynomial ring over $L$, such that $R_{L}$ is module-finite and generically étale over $A$ and such that for 
every $q=p^{e}, c R^{1 / q} \subseteq A^{1 / q}[R]:$ moreover, $A^{1 / q}[R] \cong A^{1 / q} \otimes_{A} R$ is $R$-flat for every $q$.

Proof. We note that, in essence, all of this is established in the proof of (1.5.5) of [HH6]. The fact that $\mathcal{J}(R / K)$ is not contained in a minimal prime of $R$ follows from the fact that $R$ is geometrically reduced. The statement about completely stable test elements is proved in (1.5.5) of [HH6] (there is an unnecessary additional hypothesis in [HH6] that $R$ be a domain — we discuss below why this can be removed).

The infinite field $L$ is needed so as to be able to map a polynomial ring, say in $n$ variables, onto $R$ in such a way that the variables, after a suitable linear change of coordinates, are in sufficiently general position. Then, $R$ will be a module-finite generically étale extension of any polynomial subring $A$ generated by $d$ of these variables, and it follows that every size $n-d$ minor $c$ of the matrix occurs in a Jacobian ideal $\mathcal{J}(R / A)$ (the notation agrees with that used in $\S(1.5 .2)$ of [HH6]), and so multiplies $R^{1 / q}$ into $A^{1 / q}[R]$ as a corollary of the Lipman-Sathaye Jacobian theorem [LS]. There is one point that needs a comment: the Lipman-Sathaye theorem as given in [LS] assumes that the ring $R$ is a domain, and because of this the result in [HH6] is also stated with a domain hypothesis for $R$. However, the Lipman-Sathaye theorem is valid in the reduced equidimensional case: the needed result is given in $[\mathrm{Ho}]$. Finally, we note that the isomorphism $A^{1 / q}[R] \cong A^{1 / q} \otimes_{A} R$ is proved in [HH2], Lemma (6.4), and since $A^{1 / q}$ is flat over $A$ (because $A$ is regular: cf. [Kunz]) the result follows.

Lemma 3.5. Let $R$ be a reduced Noetherian ring of positive prime characteristic $p$, let $c \in R$, and suppose that for every power $q$ of $p$ there is an $R$-flat submodule $N_{q}$ of $R^{1 / q}$ such that $c R^{1 / q} \subseteq N_{q}$. Let $W$ be a multiplicative system in $R$ and let $I$ be an ideal of $R$ that is contracted with respect to $W$. Let $I_{q}$ denote the contraction of $I^{[q]} R_{W}$ to $R$. Then for every $q=p^{e}, c^{q} I_{q} \subseteq I^{[q]}$.

Proof. Since $N_{q}$ is $R$-flat, $(R / I) \otimes_{R} N_{q}$ is $(R / I)$-flat. Since the elements of $W$ are nonzerodivisors in $R / I$, it follows that they are not zerodivisors on $N_{q} / I N_{q}$. If $u \in I_{q}$ we can choose $f \in W$ such that $f u \in I^{[q]}$ and then $f^{q} u \in I^{[q]}$ as well. Taking $q$ th roots we find that $f u^{1 / q} \in I R^{1 / q}$, and multiplying by $c$ gives that $c f u^{1 / q} \in I\left(c R^{1 / q}\right) \subseteq I N_{q}$. But $f$ is not a zerodivisor on $N_{q} / I N_{q}$ (note that $c u^{1 / q} \in N_{q}$ ) and so $c u^{1 / q} \in I N_{q} \subseteq I R^{1 / q}$. Taking $q$ th powers yields that $c^{q} u \in I^{[q]}$, as required. 
Lemma 3.6. Let $R$ be a geometrically reduced equidimensional $K$-algebra finitely generated over a field $K$ of positive prime characteristic $p$. Let $I$ be any ideal of $R$, and let $W$ be a multiplicative system consisting of nonzerodivisors modulo $I$. Let $I_{q}$ be the contraction of $I^{[q]} R_{W}$ to $R$. Then for every $q=p^{e}, \mathcal{J}(R / K)^{[q]} I_{q} \subseteq I^{[q]}$.

Proof. Let $L$ be as in Theorem 3.4. After a flat base change from $R$ to $R_{L}$ the image of $W$ still consists of nonzerodivisors on $I R_{L}$, and since $J_{R_{L} / L}=\mathcal{J}(R / K) R_{L}$ is generated by elements $c$ satisfying the condition on $c$ in the hypothesis of Lemma 3.5, if $I_{q}^{\prime}$ denotes the contraction of $I^{[q]}\left(R_{L}\right)_{W}$ to $R_{L}$, we have that $J_{R_{L} / L} I_{q}^{\prime} \subseteq I^{[q]} R_{L}$ and so $\mathcal{J}(R / K) I_{q}^{\prime} \subseteq$ ${ }^{[q]} R_{L}$. Since $I_{q} \subseteq I_{q}^{\prime}$, it follows that $\mathcal{J}(R / K) I_{q} \subseteq\left(I^{[q]} R_{L}\right) \cap R=I^{[q]}$, since $R_{L}$ is faithfully flat over $R$.

Theorem 3.7. Let $R$ be a geometrically reduced equidimensional K-algebra finitely generated over a field $K$ of positive prime characteristic $p$. Let I be any ideal such that for every prime ideal $Q$ of $R, I R_{Q}$ either contains a nonzerodivisor or else is (0) (i.e., $I$ is locally generically free). Let $h$ be the largest analytic spread of $I R_{P}$ as $P$ runs through the associated primes of $I$. Let $J=\mathcal{J}(R / K)$ be the Jacobian ideal. Then for every positive integer $n$ we have that

(a) $J^{n} I^{(h n)} \subseteq\left(I^{n}\right)^{*}($ tight closure).

(b) $J^{n+1} I^{(h n)} \subseteq I^{n}$.

More generally, for every nonnegative integer $k$ and positive integer $n$ we have that

$\left(\mathrm{a}^{\prime}\right) J^{n} I^{(h n+k n)} \subseteq\left(\left(I^{(k+1)}\right)^{n}\right)^{*}($ tight closure $)$.

$\left(\mathrm{b}^{\prime}\right) J^{n+1} I^{(h n+k n)} \subseteq\left(I^{(k+1)}\right)^{n}$.

Proof. We have stated parts (a) and (b) separately for emphasis, but evidently it suffices to prove the more general statements $\left(\mathrm{a}^{\prime}\right)$ and $\left(\mathrm{b}^{\prime}\right)$. Since $J$ consists of test elements it multiplies the tight closure of any ideal into the ideal. Thus, $\left(b^{\prime}\right)$ follows from $\left(a^{\prime}\right)$ by multiplying by $J$, and it will suffice to prove $\left(\mathrm{a}^{\prime}\right)$.

It suffices to prove the result for each connected component of Spec $R$ : tight closures may be computed componentwise, and passing to the component can be achieved by localizing at an idempotent - since formation of the Jacobian ideal commutes with localization, the new Jacobian ideal is just the expansion of the original to the factor ring corresponding to the component.

Thus, we may assume, by Lemma 2.5, that $I$ is either (0) or else contains a nonzerodivisor. In the case where $I=(0)$ there is nothing to prove. If $I$ contains a nonzerodivisor 
then this is also true for all powers of $I$. Let $u \in I^{(h n+k n)}$. Let $s$ be as in Lemma 2.4(b).

We must show that $J^{n} u \subseteq\left(\left(I^{(k+1)}\right)^{n}\right)^{*}$. Let $W$ be the complement of the union of the associated primes of $I$. For any $q=p^{e}$ we may write $q=a n+r$ with $a$ a nonnegative integer and $0 \leq r<n$, and then by Lemma 2.4(b) we have that

$$
I^{s+(h+k)(n-1)} u^{a} \subseteq\left(\left(I^{(k+1)}\right)^{[q]}\right)^{W}
$$

where the superscript ${ }^{W}$ indicates expansion to $R_{W}$ followed by contraction to $R$. Since $I^{(k+1)}$ is contracted with respect to $R_{W}$, we may use Lemma 3.6 to conclude that

$$
J^{[q]}\left(\left(I^{(k+1)}\right)^{[q]}\right)^{W} \subseteq\left(I^{(k+1)}\right)^{[q]},
$$

and so we have that

$$
J^{[q]} I^{s+(h+k)(n-1)} u^{a} \subseteq\left(I^{(k+1)}\right)^{[q]} .
$$

Taking $n$th powers and abbreviating $b=s n+(h+k)(n-1) n$ we have that

$$
I^{b}\left(J^{n}\right)^{[q]} u^{a n} \subseteq\left(\left(I^{(k+1)}\right)^{n}\right)^{[q]}
$$

for all $q$ and since $q \geq$ an this yields

$$
I^{b}\left(J^{n} u\right)^{[q]} \subseteq\left(\left(I^{(k+1)}\right)^{n}\right)^{[q]}
$$

for all $q$. Let $d$ be a fixed nonzerodivisor in $I^{b}$ (note that $b$ does not depend on $q$ ). The condition that

$$
d\left(J^{n} u\right)^{[q]} \subseteq\left(\left(I^{(k+1)}\right)^{n}\right)^{[q]}
$$

tells us precisely that $J^{n} u \subseteq\left(\left(I^{(k+1)}\right)^{n}\right)^{*}$, as required.

Example 3.8. Consider the ring $R=K[x, y, z] /\left(x y-z^{n}\right)$. The Jacobian ideal $J=$ $\mathcal{J}(R / K)$, if $n$ is a unit, is $\left(x, y, z^{n-1}\right) R$. Let $P=(y, z)$. Then $h=1$, and $y \in P^{(n)}$. Then $J^{n-1}$ multiplies $y$ into $P^{n}$ but no smaller power does since $y \in J$ and $y^{n-2} y \notin P^{n}$. This suggests that the result in 3.7 (take $I=P$ ) is close to best possible. We do not know, however, whether the exponent $n$ used in parts (a) and $\left(\mathrm{a}^{\prime}\right)$ can be replaced by $n-1$ in general. Of course, if so, then the exponent $n+1$ can be replaced by $n$ in parts (b) and $\left(b^{\prime}\right)$. 


\section{The equal characteristic zero case}

We now give the extensions of the various positive characteristic results to the equal characteristic case. As mentioned in the introduction, the notion of tight closure that we use here is that of equational tight closure from [HH6, $\S 3.4 .3-4]$. The main results of this section are contained in Theorem 4.4 below. We need to do some groundwork before we can prove that theorem, however. The proof of the main results depends on three steps: one is to localize and complete, the second is to descend from the complete case to the affine case, and the third is to use reduction to positive characteristic in the affine case. The second step is based on the following result from $[\mathrm{AR}]$ :

Theorem 4.1. Let $K$ denote either a field or an excellent discrete valuation ring. Let $T=K\left[\left[x_{1}, \ldots, x_{n}\right]\right]$ be the formal power series ring in $n$ variables over $K$. Then every $K$ algebra homomorphism of a finitely generated $K$-algebra $R$ to $T$ factors $R \rightarrow S \rightarrow T$ where

the maps are $K$-algebra homomorphisms and $S$ has the form $\left(K\left[x_{1}, \ldots, x_{n}, y_{1}, \ldots, y_{t}\right]_{m}\right)^{\mathrm{h}}$, where the $x_{i}$ are as above, the $x_{i}$ and $y_{j}$ are algebraically independent elements, over $K$, of the maximal ideal of $T, m$ is the ideal of the polynomial ring $K[x, y]$ generated by $(x, y)$ and, if $K$ is a DVR, by the generator of the maximal ideal of $K$, and $\mathrm{h}$ denotes Henselization.

This is a special case of general Néron desingularization (cf. [Po1], [Po2], [Og], [Swan]), but the argument is simpler in this case (we note that [Swan] has removed any possible doubt about the validity of the general theorem - however, we only need the result of $[\mathrm{AR}])$. In [HH6] this is used to prove the following result, which is Theorem (3.5.1) there:

Theorem 4.2. Let $K$ be a field of characteristic zero and let $(S, m, L)$ be a complete local ring that is a $K$-algebra. Assume that $S$ is equidimensional and unmixed.

Suppose that $R_{0}$ is a subring of $S$ that is finitely generated as a $K$-algebra. We also assume given finitely many sequences of elements $\left\{z_{t}^{(i)}\right\}_{t}$ in $R_{0}$, each of which is part of a system of parameters for $S$.

Then there is a finitely generated $K$-algebra $R$ such that the homomorphism $R_{0} \hookrightarrow S$ factors $R_{0} \hookrightarrow R \rightarrow S$ and such that the following conditions are satisfied:

(1) $R$ is biequidimensional.

(2) The image of each sequence $\left\{z_{t}^{(i)}\right\}_{t}$ in $R$ is a sequence of strong parameters: this 
means that after localization and completion at any prime that contains them, they form part of a system of parameters modulo every minimal prime.

(3) If $\mathbf{m}$ is the contraction of $m$ to $R$, then $\operatorname{dim} R_{\mathbf{m}}-\operatorname{depth} R_{\mathbf{m}}=\operatorname{dim} S-\operatorname{depth} S$. In particular, $R_{\mathbf{m}}$ is Cohen-Macaulay iff $S$ is Cohen-Macaulay.

(4) If $S$ is a reduced (respectively, a domain) then so is $R$.

(N.B. In general, $\operatorname{dim} R_{m}$ is substantially bigger than $\operatorname{dim} S$.)

In the sequel we need a version of this result in which the equidimensionality of the ring is not assumed. Moreover, we need to keep track of some complexes of modules, bounds on analytic spreads after localization at associated primes, the fact that one ideal is a certain symbolic power of another, and so forth. The following result suffices:

Theorem 4.3. Let $K$ be a field of characteristic zero and let $(S, m, L)$ be a complete local ring that is a K-algebra. Assume given each of the following:

(1) Finitely many finitely generated $S$-modules with specific finite presentations, finitely many maps of these $S$-modules with specific presentations of the maps, and finitely many specified equalities among the compositions of these maps.

(2) Using the modules and maps in (1), finitely many short exact sequences. Finitely many finite complexes with specified homology. Finitely many instances in which one of the specified modules is identified with a submodule of another. Finitely many instances in which one of the modules is specified to be the intersection of finitely many of the others, where all are submodules of a given specified module.

(3) Finitely many ideals of $S$ with specified generators.

(4) From among the ideals in (3), a finite subset with a finite set of associated primes of specified heights, and a finite subset such that the maximum analytic spread after localizing at an associated prime has a given bound.

(5) From among the ideals in (4), finitely many choices of $I, I^{\prime}$, such that $I$ and $I^{\prime}$ have the same associated primes. Also, finitely many choices of $I$ and $I^{\prime}$ and an integer $k$ such that $I^{\prime}=I^{(k)}$.

(6) From among the ideals and modules given in (1) and (3), finitely many pairs $M$, $I$ such that $I M=0$.

(7) A finite set of finite sequences in $S$, each of which is specified to be part of a system of parameters for $S$, and a finite set of sequences each of which is specified to be a regular sequence on a given one of the given modules. 
(8) A finitely generated $K$-subalgebra $R_{0} \subseteq S$ so large that it contains all the entries needed for the presentations of the modules and maps in (1), all of the specified generators of the ideals in (3), and the elements of the sequences in (7), so that we may view all of the given modules, maps, sequences, and ideals as arising from corresponding ones over $R_{0}$ either by tensoring, taking images, or expanding ideals.

Then there is a finitely generated $K$-algebra $R$ such that the homomorphism $R_{0} \hookrightarrow S$ factors $R_{0} \hookrightarrow R \rightarrow S$ and such that the following conditions are satisfied:

(a) The specified presentations of maps of modules are maps of modules over $R$, and the specified exacts sequences of modules, descended to $R$ by tensoring up from the their counterparts over $R_{0}$, are exact. All of the other specified relations among the given modules and ideals continue to hold after descent, including specifications of the homology of a given complex and specifications that a certain submodule (or ideal) be a finite intersection of finitely many given other submodules (or ideals). Likewise, the specification that a certain ideal be the annihilator of a certain module can be preserved.

(b) The image (under the map $R_{0} \rightarrow R$ ) of each set of elements that is part of a system of parameters for $S$ has height equal to its length. The image of each regular sequence on a specified module is a regular sequence on the corresponding module over $R$.

(c) The specified ideals, descended to $R$ by expanding their counterparts over $R_{0}$, are unmixed when the original ideals are. For a specified ideal I, the greatest number of generators and the greatest analytic spread after localization at an associated prime do not increase. Moreover, for the given choices of $I, I^{\prime}, k$ such that $I^{\prime}=I^{(k)}$, this remains true after descent to $R$.

(d) $R$ is regular if $S$ is.

(N.B. In general, $\operatorname{dim} R_{m}$ is substantially bigger than $\operatorname{dim} S$.)

Proof. If $S$ is regular we apply the Artin-Rotthaus theorem (4.1) directly to the power series ring $S=L\left[\left[x_{1}, \ldots, x_{n}\right]\right]$, where the coefficient field $L$ has been chosen to contain $K$. We first solve the problem over $L$ and then descend to $K$. As the latter step is routine, we shall simply treat the case $L=K$.

We are free to enlarge $R_{0}$ repeatedly, and so may assume that $x_{1}, \ldots, x_{n} \in R_{0}$. Since the Henselization of a local ring is a direct limit of finitely presented étale extensions, 
we have that $S$ is a filtered inductive limit of regular rings $R$ of finite type over $L$ with maps $R_{0} \rightarrow R \rightarrow S$ such that $R$ is smooth over $L\left[x_{1}, \ldots, x_{n}\right]$ and such that the $x_{i}$ form a permutable regular sequence in $R$. In this case, we can keep track of whether a sequence of elements is part of a system of parameters by extending it to a full system of parameters, say, $y_{1}, \ldots, y_{n}$. There will be equations expressing a power of every $y_{j}$ as a linear combination of the $x_{i}$ and conversely. We may enlarge $R_{0}$ so that all these equations hold in $R_{0}$. Conditions such as having a specified ideal kill a specified module are likewise expressible equationally and can be guaranteed by enlarging $R_{0}$. We shall leave many straightforwarded details to the reader, noting that the proof of (3.5.1) in [HH6] is given in great detail and is a very similar kind of argument. We focus here only on some critical issues.

One can keep track of short exact sequences by using an exact sequence of finite free resolutions over $R$. The rows will be split exact. To guarantee that a finite free resolution stays a resolution one keeps track of all the matrices. $R_{0}$ is enlarged to contain all their entries. The condition that one has a complex is equational, and so is the condition that the determinantal ranks be preserved. By the result of [BE], one only needs to guarantee that the largest nonvanishing ideals of minors have specified depths, i.e., that each contains a subset, of a certain specified size, of a system of parameters for the ring, and we may apply the discussion of the preceding paragraph.

This enables one also to keep track of finite complexes with specified homology (express all the conditions by using suitable short exact sequences) and of finite intersections as well: e.g., the intersection of $N_{1}$ and $N_{2}$ within $N$ may be characterized as the kernel of the map $N \rightarrow N / N_{1} \oplus N / N_{2}$. The annihilator $I$ of a single element $u$ of a module $N$ may be characterized by an injection $R / I \rightarrow N$ carrying the image of 1 to $u$, and the annihilator of $N$ may be characterized as the intersection of the annihilators of specified generators of $N$.

One can preserve depths of modules and, hence, regular sequences by expressing them in terms of the vanishing of Koszul homology.

One can keep the associated primes of an ideal having specified heights as follows: keep track of its entire primary decomposition, preserving the fact that components intersect to give the ideal. To preserve the relation between an ideal $I$ primary to $P$ and and $P$, note that $S / I$ has a filtration by modules that are embeddable in finitely generated free 
$(S / P)$-modules, and this can be preserved. Now, the height property can be preserved by keeping $P$ height unmixed in the descent: we do not need to keep $P$ prime. This can be achieved by writing $P$ in the form

$$
\left(f_{1}, \ldots, f_{d}\right) R:_{R} g R
$$

where the elements $f_{i}$ are part of a system of parameters for $S$. Note that the number of primary components may increase, but the largest height of an associated prime does not. The same idea can be used to ensure that two specified ideals $I, I^{\prime}$ that have the same associated primes continue to do so.

When $S$ is not necessarily regular write it as $T / J_{0}$ where $T$ is regular, and transfer the problem to $T$ (while keeping track of $J_{0}$ ). Ideals of $S$ correspond to ideals of $T$ that contain $J_{0}$, and $R$-modules to $T$-modules that are killed by $J_{0}$. For example, to maintain a specific symbolic power relationship, one may suppose that one of the ideals is $I \supseteq J_{0}$ and that it has a certain set $\mathcal{S}$ of associated primes while the other has the form $\left(I^{k}+J_{0}\right): g$ with $g$ a nonzerodivisor on $I$ and that it has as its associated primes a certain subset of $\mathcal{S}$. We have seen that all this can be preserved while descending.

Finally, we want to explain how to preserve the condition that the maximum analytic spread of $I$ after localizing at an associated prime of $I$ be at most $h$. Again, we think of $S$ as $T / J_{0}$ where $T$ is regular. Call the associated primes $P_{1}, \ldots, P_{s}$. Then for each $P_{i}, I S_{P_{i}}$ is integral over $I R_{P_{i}}$ after localizing at $P_{i}$. Thus, for each $P_{i}$ we can choose an element $v=v_{i}$ not a zerodivisor on $P_{i}$ such that $I R_{v}$ is integral over $I_{0} R_{v}$, where $I_{0}$ is generated by at most $h$ elements of $R$. (In the equal characteristic 0 case, the residue field is always infinite.) After clearing denominators by multiplying by a power of $v$, for each generator $r$ of $I$ we get an equation

$$
v^{N} r^{t}+i_{1} r^{t-1}+\cdots+i_{k} r^{k}+\cdots+i_{t-1} r+i_{t}=j
$$

with $i_{k} \in I_{0}^{k}$ and with $j \in J_{0}$. We can preserve all this by placing all of the needed elements in $R_{0}$. As we descend, the $P_{i}$ are replaced by unmixed ideals, while each $v_{i}$ is kept a nonzerodivisor on the descended version of $P_{i}$. By including sufficiently many coefficients in $R_{0}$ we can preserve that every $i_{k} \in I_{0}^{k}$ where $I_{0}$ is generated by at most $h$ elements. Any associated prime of the descended version of $I$ will be an associated prime of one of the descended $P_{i}$, and so will fail to contain the corresponding $v_{i}$. Thus, after 
descent, when one localizes at an associated prime of the descended version of $I$, at least one of the $v_{i}$ becomes invertible, and it follows that the descended version of $I$ becomes integrally dependent on an ideal with at most $h$ generators.

Theorem 4.4. Let $R$ be Noetherian ring containing a field of characteristic 0 . Let $I$ be any ideal of $R$, and let $h$ be the largest analytic spread of $I R_{P}$ for $P$ an associated prime of $I$.

(a) If $R$ is regular, $I^{(h n)} \subseteq I^{n}$ for all positive integers $n$. More generally, $I^{(h n+k n)} \subseteq$ $\left(I^{(k+1)}\right)^{n}$ for all positive integers $n$ and nonnegative integers $k$.

(b) If I has finite projective dimension then $I^{(h n)} \subseteq\left(I^{n}\right)^{*}$ for all positive integers $n$.

(c) If $R$ is affine and equidimensional over a field $K$, and locally $I$ is either 0 or contains a nonzerodivisor, then with $J=\mathcal{J}(R / K)$, for every nonnegative integer $k$ and positive integer $n$ we have $J^{n} I^{(h n+k n)} \subseteq\left(\left(I^{(k+1)}\right)^{n}\right)^{*}$ and $J^{n+1} I^{(h n+k n)} \subseteq$ $\left(I^{(k+1)}\right)^{n}$. In particular, $J^{n} I^{(h n)} \subseteq\left(I^{n}\right)^{*}$ and $J^{n+1} I^{(h n)} \subseteq I^{n}$ for all $n$.

Proof. We first prove (c), and at the same time we prove (b) for finitely generated algebras over a field $K$. We use the standard descent theory of Chapter 2 of [HH6] to replace the field $K$ by a finitely generated $Z$-subalgebra $A$, so that we have a counterexample in an affine algebra $R_{A}$ over $A$ with $R_{A} \subseteq R$ and $R \cong K \otimes_{A} R_{A}$. In particular, $R_{A}$ will be reduced. In doing so we descend $I$ to an ideal $I_{A}$ of $R_{A}$ as well as the ideals and their prime radicals in its primary decomposition. We have an element $u_{A}$ that fails to satisfy the containment we are trying to prove. In the regular case, we can localize at a nonzero element of $A$ to make $R_{A}$ smooth over $A$. In either case, we can localize at a nonzero element of $A$ to make $A$ smooth over $\mathbb{Z}$. Since $\mathcal{J}\left(R_{A} / A\right) \mathcal{J}(A / \mathbb{Z}) \subseteq \mathcal{J}\left(R_{A} / \mathbb{Z}\right)$ and since $\mathcal{J}(A / \mathbb{Z})=A$ when $A$ is smooth over $\mathbb{Z}$, we see that we may assume that $\mathcal{J}\left(R_{A} / A\right) \subseteq \mathcal{J}\left(R_{A} / \mathbb{Z}\right)$, which means that we can work over $\mathbb{Z}$ instead of $A$. The result now follows from the fact that, for almost all fibers, the containment holds for the map $\mathbb{Z} \rightarrow R_{A}$ after passing to fibers over closed points of Spec $\mathbb{Z}$. Notice that as we pass to fibers $\kappa \rightarrow R_{\kappa}$ we may assume that each minimal prime $P_{A}$ of $I_{A}$ becomes a radical ideal whose minimal primes in $R_{\kappa}$ are all of the same height as the original. Thus, in the fiber, the primary decomposition of $I_{\kappa}$ may have more components, but each of these will be obtained from the image of one of the original components by localization. The biggest analytic spread after localizing at an associated prime will not change. It follows in both parts that we have the required containment in a tight closure. In the regular case, we have that all ideals are tightly closed. 
We now consider the general case for (a) and (b). The problem in each part reduces to the local case: note that it suffices to check whether an element is in a tight closure locally after completion.

One may then complete: although $I \widehat{R}$ may have more associated primes, Discussion 2.3 (c) shows that the biggest analytic spread as one localizes at these cannot increase. Note that once we have $\widehat{I}^{(h n)} \subseteq\left(\widehat{I}^{n}\right)^{*}$ in $\widehat{R}$, it follows that $I^{(h n)} \subseteq\left(\left(I^{n}\right) \widehat{R}\right)^{*}$ since $\widehat{R}$ is flat over $R$, and this implies that $I^{(h n)} \subseteq\left(I^{n}\right)^{*}$, which is $I^{n}$ when $R$ is regular.

In the regular case, next note that $\widehat{I}^{(k+1)}=I^{(k+1)} \widehat{R}$, so that $\left(\widehat{I}^{(k+1)}\right)^{n}=\left(I^{(k+1)}\right)^{n} \widehat{R}$. (The associated primes of $\widehat{R} / I^{(k+1)} \widehat{R}$ are among those associated to $\widehat{R} / P \widehat{R}$ for some associated prime $P$ of $I$, by Proposition 15 in IV B.4. of [Se], since any associated prime of $I^{(k+1)}$ must be an associated prime of $I$, and by another application of Proposition 15 in IV B.4. of [Se] these in turn are associated primes of $I \widehat{R}$.) Thus, we get

$$
I^{(h n+k n)} \subseteq \widehat{I}^{(h n+k n)} \subseteq\left(\widehat{I}^{(k+1)}\right)^{n}=\left(I^{(k+1)}\right)^{n} \widehat{R}
$$

and so

$$
I^{(h n+k n)} \subseteq\left(I^{(k+1)}\right)^{n} \widehat{R} \cap R=\left(I^{(k+1)}\right)^{n},
$$

as required, by the faithful flatness of $\widehat{R}$ over $R$.

Using Theorem 4.3 above one may then descend to a suitable affine algebra over a coefficient field for the complete local ring, and the results follow from what we have already proved in the affine case.

\section{Questions}

Evidently, if we fix an ideal $I$ in a Noetherian ring $R$, for every integer $N$ there is a least integer $g(N) \in \mathbb{N}$ such that $I^{(g(N))} \subseteq I^{N}$, and there are clearly deep results about the behavior of $g(N) / N$. Our result that in equicharacteristic regular rings (or when $I$ has finite projective dimension), $g(N) / N$ is bounded by the largest height of an associated prime of $I$ (or the largest analytic spread of $I R_{P}$ for $P$ an associated prime of $I$ ) might be improved in any number of ways.

One possibility is to study the case where the ring need not be regular (and $I$ does not have finite projective dimension). Note that our results here for this case, involving the 
Jacobian ideal, do not directly give information about the question raised just above. (We mention again that we do not know whether the exponents used on the Jacobian ideal in Theorem 3.7 and Theorem 4.4(c) are best possible: it may be possible to decrease the exponent by 1.)

We do not know what the situation is in mixed characteristic regular rings. But even in equicharacteristic regular rings there may be better bounds that make use of additional information about $I$. Notice, for example, that the height is never the best bound when $I$ is $m$-primary in a regular local ring $(R, m)$, since then $I^{(n)}=I^{n}$ for all $n$.

It is not clear what the best bound is in equicharacteristic regular rings even for primes of codimension 2 .

In quite a different direction, we observe that there have been several instances in which the theory of multiplier ideals and tight closure theory have either interacted, or have been used to prove similar results. E.g., tight closure can be used to prove the Briançon-Skoda theorem (cf. [HH2, §5]), as can the theory of multiplier ideals. (Cf. [L2], where these are called adjoint ideals. This is also done implicitly in [EL] and explicitly in [Laz, §10]). The connection between the multiplier ideal and the test ideal of tight closure theory is explored in [Sm2]. It would be desirable to understand fully the underlying reasons for this connection.

\section{BIBLIOGRAPHY}

[ArR] M. Artin and C. Rotthaus, A structure theorem for power series rings, in Algebraic Geometry and Commutative Algebra: in honor of Masayoshi Nagata, Vol. I, Kinokuniya, Tokyo, 1988, pp. 35-44.

[BE] D. Buchsbaum and D. Eisenbud, What makes a complex exact, J. of Algebra 25 (1973), 259-268.

[EL] L. Ein and R. Lazarsfeld, A geometric effective Nullstellensatz, Invent. Math. 137 (1999), 427448.

[ELS] L. Ein, R. Lazarsfeld, and K. Smith, Uniform bounds and symbolic powers on smooth varieties, Invent. Math. 144 (2001), 241-252.

[Her] J. Herzog, Ringe der Characteristik $p$ und Frobenius-funktoren, Math Z. 140 (1974), 67-78.

[HH1] M. Hochster and C. Huneke, Tight closure and strong F-regularity, Mémoires de la Société Mathématique de France, numéro 38 (1989), 119-133.

[HH2] - Tight closure, invariant theory, and the Briançon-Skoda theorem, J. Amer. Math. Soc. 3 (1990), 31-116.

[HH3] _ F-regularity, test elements, and smooth base change, Trans. Amer. Math. Soc. 346 (1994), 1-62.

[HH4] Infinite integral extensions and big Cohen-Macaulay algebras, Annals of Math. 135 (1992), 53-89.

[HH5] Applications of the existence of big Cohen-Macaulay algebras, Advances in Math. 113 (1995), 45-117. 
[HH6] - Tight closure in equal characteristic zero, preprint.

[Ho] M. Hochster, Presentation depth and the Lipman-Sathaye Jacobian theorem, to appear, Homology, Homotopy and Applications (issue in honor of Jan-Erik Roos), International Press, Cambridge, MA.

[Hu] C. Huneke, Tight Closure and Its Applications, Proc. of the CBMS Conference held at Fargo, North Dakota, July, 1995, C.B.M.S. Regional Conference Series, Amer. Math. Soc., Providence, R.I., 1996.

[Kunz] E. Kunz, Characterizations of regular local rings of characteristic p, Amer. J. Math. 91 (1969), $772-784$.

[Laz] R. Lazarsfeld, Multiplier ideals for algebraic geometers, preliminary version of part of a book, available at the site http://www.math.lsa.umich.edu/ rlaz/.

[Ł1] J. Lipman, Relative Lipschitz saturation, Amer. J. Math. 97 (1975), 791-813.

[Ł2] - Adjoints of ideals in regular local rings, Math. Res. Lett. 1 (1994), 739-755.

[LS] J. Lipman and A. Sathaye, Jacobian ideals and a theorem of Briançon-Skoda, Michigan Math. J. 28 (1981), 199-222.

[NR1] D. G. Northcott and D. Rees, Reductions of ideals in local rings, Proc. of the Cambridge Phil. Soc. 50 (1954), 145-158.

[NR2] D. G. Northcott and D. Rees, A note on reductions of ideals with an application to the generalized Hilbert function, Proc. of the Cambridge Phil. Soc. 50 (1954), 353-359.

[Og] T. Ogoma, General Néron desingularization based on the idea of Popescu, J. of Algebra 167 (1994), 57-84.

[Po1] D. Popescu, General Néron desingularization, Nagoya Math. J. 100 (1985), 97-126.

[Po2] - General Néron desingularization and approximation, Nagoya Math. J. 104 (1986), 85115.

[PS] C. Peskine and L. Szpiro, Dimension projective finie et cohomologie locale, Publ. Math. I.H.E.S. (Paris) 42 (1973), 323-395.

[Se] J.-P. Serre, Algèbre local - Multiplicités, Lecture Notes in Mathematics 11, Springer-Verlag, Berlin, Heidelberg, New York, 1965.

[Sm1] K. E. Smith, Tight closure of parameter ideals, Invent. Math. 115 (1994), 41-60.

[Sm2] K. E. Smith, The multiplier ideal is a universal test ideal, Communications in Algebra 28 (2000), $5915-5929$.

[Swan] R. Swan, Néron-Popescu Desingularization, expanded notes from a Univ. of Chicago series of lectures in Spring, 1995, $42 \mathrm{pp}$.

[Swsn] I. Swanson, Linear equivalence of topologies, Math. Z. 234 (2000), 755-775.

\author{
Department of MATHEMATiCs \\ UNIVERSITY OF MICHIGAN \\ ANN ARBOR, MI 48109-1109 \\ USA \\ E-MAIL: \\ hochster@umich.edu
}

\author{
Department of MATHEMATiCs \\ UNIVERSITY OF KANSAS \\ LAWRENCE, KS 66045 \\ USA \\ E-MAIL: \\ huneke@math.ukans.edu
}

